\title{
Ganas de Emprender y Felicidad: un Estudio Exploratorio a Partir del Global Entrepreneurship Monitor en Chile.
}

\author{
Sergio Hinojosa Martínez', Carlos Albornoz Pardo²
}

\begin{abstract}
This study focuses on the determinants that lead individuals to have the desire to entrepreneur, in which happiness is included. It draws on the social psychology literature and applies the theory of planned behavior to understand and predict entrepreneurial desire. To test the integrity of this theory in predicting entrepreneurial behavior, this study uses data from the Global Entrepreneurship Monitor (GEM) research program in Chile. Previous investigations have studied the role of happiness indirectly, not as an independent variable. Accordingly, inspired by the recommendation of Casrud \& Brannback (20II), and also the suggestion of Elfing et al. (2009) of adjusting the Shapero's (1982) standard model of the Entrepreneurial Event (SEE), in this study happiness is introduced both as specific as moderating variable, which is estimated using a logit model. The findings of the study show that happiness has a negative relationship with entrepreneurial desire. Thus, the greater the happiness, the less likely to have a desire to entrepreneur. Implications of these findings are discussed.

En este documento se utilizan datos del Global Entrepreneurship Monitor 2012 (GEM) para estimar empíricamente los determinantes de la Intención de Emprender (IE) en Chile, entre los que se incluye la felicidad. Trabajos previos han estudiado indirectamente, y de manera muy general, el rol de la felicidad en la IE, sin incorporarla como una variable explicativa. En consecuencia, inspirados en las recomendaciones de Casrud y Brannback (20II), y en la invitación de Elfing et al. (2009) de ajustar el modelo estándar de IE [Shapero (1982) y Ajzen (1991)], en este trabajo se introduce la felicidad tanto como una variable específica como moderadora, la cual se estima a través de un modelo LOGIT. Los resultados del modelo muestran que la felicidad se relaciona de manera inversa con la Intención de Emprender. De esta forma, en la medida que es mayor la felicidad, menor es probabilidad de intención de emprender (o viceversa).
\end{abstract}

Keywords: happiness; entrepreneurship desire; theory of planned behavior; global entrepreneurship monitor; logit model. intención de emprender; felicidad; modelo logit.

Universidad del Desarrollo. Av. La Plaza, 680, Las Condes. Santiago, Chile. Phone: 0223279205.

Email: 'shinojosam@udd.cl, ${ }^{2}$ calbornoz@udd.cl.

ISSN: 07 I8-2724. (http://www.jotmi.org)

Journal of Technology Management \& Innovation (c) Universidad Alberto Hurtado, Facultad de Economía y Negocios. 


\section{Introducción}

Hasta antes del trabajo inicial de Easterlin (1974), la felicidad como concepto se estudiaba principalmente en las disciplinas de la sicología y la filosofía. En la primera se utilizaba el concepto de bienestar subjetivo y en la segunda el concepto de felicidad propiamente tal. Sin embargo, a partir del año 1974 se ha empezado a estudiar de manera creciente en distintas y variadas disciplinas como: biología, ciencias políticas, management y economía por nombrar algunas. En efecto, desde esa fecha han aparecido una serie de artículos académicos que veremos en el transcurso de esta investigación. Las directrices de estos artículos han sido estudiar los efectos de la felicidad en la economía. También se abocan a los determinantes de la felicidad y sus impactos de distintos ámbitos de la vida de las personas junto con su proceso de toma de decisiones.

El año 2000 se lanzó el primer ejemplar del Journal of Happiness Studies que incluye de manera mensual temas relacionados con la evaluación cognitiva del buen vivir. Destacan temas tales como: satisfacción por la vida, el disfrute de la vida controlado por rasgos de personalidad, evaluación de la vida, entre otros. De una revisión de los últimos volúmenes disponibles del presente año, es posible encontrar artículos relacionados con el costo de la felicidad [Binder y Broekel (20/2)], la espiritualidad como elemento esencial para el bienestar personal [Van Dierendonk (2012)], predicciones de la felicidad en adolecentes [Butkovic et al. (20I2)] y el rol del gobierno en la felicidad de las personas [Kim y Kim (20I2)]. Se reafirma entonces de que hay evidencia que se está estudiando la felicidad de manera científica.

El tópico de la felicidad no sólo ha estado activo en el mundo estrictamente académico. En efecto, en especial en los últimos años, en Estados Unidos se ha escrito una cantidad enorme de libros "populares" [Acher (2010), Rubin (20II), Grinde (20I2)], cuyo principal mensaje es la felicidad: como entenderla y como alcanzarla.

Martin Seligman \{es considerado el Padre de la Sicología Positiva, rama de la sicología que estudia las emociones placenteras, el desarrollo de las virtudes y la búsqueda de la felicidad\} recientemente ha escrito un libro que se está transformando en un best seller en Estados Unidos denominado "Flourish: A Visionary New Understanding of Happiness and Well-being (20I2)" \{solamente, para mostrar la vasta literatura "popular" que se ha escrito sobre felicidad, una búsqueda rápida en la sección libros de www.amazon. com por la palabra "happiness" es posible encontrar 28.673 libros\}. Al respecto, y destacando el interés del público sobre el entendimiento de la felicidad, el año 2005 Claudia Wallis, de la revista Time, publicó un artículo de portada sobre "The New Science of Happiness", destacando la cre- ciente cantidad de información y estudios que han emergido sobre el tema.

El rol de la felicidad en la vida de las personas también está siendo fuertemente promovido por los principales economistas y líderes de opinión empresariales. El año 2005, el profesor Richard Layard ffundador de la London Business School\} publicó un libro titulado "La felicidad: Lecciones de una nueva ciencia" invitando firmemente a un cambio en la mentalidad global acerca de como mirar la economía y de esta forma cambiar la orientación desde el materialismo por la felicidad.

La sociedad ha empezado a apreciar el creciente cuerpo de investigación de la felicidad, que demuestra que a medida que las personas se vuelven más felices se hacen más saludables, energéticos, productivos, creativos, cooperativos, compasivos, e incluso más emprendedores [Unanue (2012)]. El año 2006, el curso más popular en la Universidad de Harvard fue sobre la felicidad, cuyo sello era que el camino a la felicidad no se construye mirando el PIB de un país, según el profesor de psicología positiva Tal Ben Shahar (20I2).

Posteriormente en el año 2007, el tema de la felicidad alcanzó un amplio público a nivel mundial, en la que Malcolm Gladwell describe en su libro "The Tipping Point" de manera elegante. Se trata a la felicidad como presagio de cambios importantes en nuestras experiencias personales y sociales relacionados con el buen vivir y el bienestar subjetivo.

A partir de la contribución de Gladwell, quien combina economía y psicología, se incluye el análisis de la felicidad como parte de la Economía del Comportamiento (Behavioral Economics). Este nuevo campo de estudio se orienta a entender los juicios y el proceso de toma decisión de las personas, usando factores cognitivos, sociales y emocionales. La rama de la Economía del Comportamiento ha estado liderada por Dan Ariely en su libro seminal "Predictably Irrational (2009)". Richard Thaler y Cass Sunstein, el año 2009 publican el libro "Nudge" \{traducido como "empunjoncitos"\}. En su argumento central, los autores cuestionan el efecto de las decisiones de los individuos en el bienestar, demostrando cómo la arquitectura de la elección puede exitosamente generar nudges. El propósito de estos "empujoncitos", es hacer que los individuos tomen mejores decisiones desde el ámbito de la salud, la riqueza, la felicidad y el emprendimiento.

Por otra parte, el emprendimiento, entendido como la creación de una organización capaz de capturar una oportunidad de negocio, se ha consolidado como una de las disciplinas que mas atención ha recibido en el campo de los negocios. Una de las causas que ha llevado a los gobiernos y académicos a interesarse cada vez más con mayor interés 
en el emprendimiento, es la creciente evidencia que relaciona emprendimiento y crecimiento económico [Baumol (1993); Acs et al. (2004), Larroulet y Couyoumdjian (2009)]. Según Baron y Shane (2008) y Ethugala (20I I), la creación de nuevos negocios es considerado el "combustible" del crecimiento económico. También existe una creciente literatura en cuanto al impacto del emprendimiento en el desarrollo económico [Amorós y Cristi (201 I)]. Aun cuando la cantidad de estudios para comprender por qué un individuo se decide a iniciar un negocio ha ido en aumento, todavía se encuentra una amplia controversia en la literatura sobre cuales son las causas que llevan a una persona a emprender. Una de las variables que está ganando creciente atención es la felicidad.

Carree y Verheul (2012) publicaron recientemente un estudio que explica qué hace felices a los emprendedores. Su conclusión es que los individuos con mayor nivel de capital humano tienen mayores niveles de felicidad cuando perciben un mayor nivel de ingresos. Esto se cumple una vez que se alcanza una etapa de madurez en el negocio emprendido. Los autores agregan que las mujeres siente mayor felicidad que los hombres si el emprendimiento es exitoso, no obstante, el nivel de ingreso puede ser menor que el de los hombres. Profundizar la comprensión sobre el rol que tiene la felicidad en la decisión de emprender es uno de los propósitos de este estudio. Por la creciente importancia de la felicidad en el proceso de toma de decisiones de las personas y por el efecto que tiene el bienestar subjetivo en el diseño e impactos de las políticas públicas, junto con el rol que tiene el emprendimiento en el crecimiento económico, en este documento interesa estudiar el mecanismo de transmisión de la felicidad en la intención de emprender.

\section{Marco Conceptual}

Si bien sólo a partir de los 90' es posible encontrar literatura científica sobre qué es y cómo se produce la felicidad, los esfuerzos por comprender la felicidad son bastante antiguos. Ya en la antigua Grecia, Aristóteles sugería entender la felicidad como el significado y propósito de la vida, siendo el objetivo último de la existencia humana \{ver Texto 13 Felicidad: la Idea Aristotélica de la Felicidad Humana Entendida Como la Vida de Acuerdo a la Mente. Aristóteles, Ética a Nicómaco, X\}. En Chile, la Constitución de 1818 mandata al gobierno de manera obligatoria a proporcionar el camino de la felicidad y la prosperidad \{ver Biblioteca del Congreso Nacional de Chile. www.ben.cl\}.

Si recurrimos a la esencia económica del problema de la escasez [Keynes (1936)], es posible asumir que el problema de la economía es cómo ocupar el tiempo de ocio para vivir sabiamente y en armonía con los demás, una vez superada la escasez material. Pasado un umbral, no tiene sentido preo- cuparse sólo por los elementos materiales: hay que preocuparse por otros aspectos que también inciden en la felicidad. Esa es la preocupación central de lo que algunos llaman la nueva ciencia de la felicidad [Binder 2006)].

La felicidad es también una creciente preocupación entre algunos economistas. Una de las contribuciones importantes de la economía al estudio de la felicidad ha estado en el desafío de cómo medir esta variable. Por mucho tiempo, los economistas han creído en modelos de preferencias reveladas [Alesina et al. (2004)] y no de preferencias reales ("puedo decir que soy feliz, pero en verdad soy infeliz"). El debate al interior de la ciencia económica en torno a si es posible medir la felicidad ha generado una vasta literatura sobre el tema [Binder (20/2)].

En términos generales se puede decir que hay tres grandes postulados en torno a la felicidad. El primero de ellos tiene que ver con la Paradoja de Easterlin [Easterlin (1974)], la cual incentiva el debate de eliminar el PIB como el "gran" indicador que gobierna las políticas económicas, puesto que demuestra que el aumento del ingreso no hace más feliz a la gente: la felicidad no se compra. Se observa empíricamente que al comparar entre países, los más ricos no son más felices que los pobres. En el tiempo, el aumento del ingreso no se ve reflejado en el aumento de felicidad por habitante. Sin embargo dentro de un mismo país, los más ricos son más felices que los pobres. En contraste, nuevos estudios con modelos econométricos más precisos con data actualizada llegaron a demostrar que esta paradoja no necesariamente se cumple [Stevenson y Wolfers (2008)].

El segundo postulado es conocido como la adaptación (ajuste) de aspiraciones ó la teoría del set point [Lykken (1996)] la cual plantea que habría un mayor nivel de satisfacción, de felicidad que viene determinada por factores genético \{también hay estudios recientes que muestran que la intención de emprender de los individuos tiene raíces genéticas. Ver Nicolau y Shane (2009), (2010), Chaturvedi et al. (20II) y Laspita et al. (20I2)\}, por los cambios en las circunstancias que desvían a las personas. Es decir, la teoría indica que los seres humanos van ajustando sus aspiraciones a medida que son más felices, puesto que buscan más felicidad, $y$ a medida que van buscando, la felicidad siempre vuelve al nivel básico o mismo punto de partida. Nunca se llega a ningún parte porque siempre se van ajustando las expectativas. Diener et al. (2009) lo asemejan a que los individuos estuvieran cambiando el objetivo como en una trotadora [moving target, treadmill].

En la teoría del set point, se pueden mirar los niveles de las relaciones en distintos contextos, como por ejemplo personas que viven en ambientes complicados son más felices por que saben y valoran lo poco que tienen. Por ejemplo, 
Graham y Shapiro (20I2) señalan que los afganos son tan felices como los latinos, y $20 \%$ más proclives a sonreír que los cubanos. Así, ambos autores corroboran la teoría de la adaptación, ampliándola a una nueva interpretación cultural de la felicidad, sobre como entenderla en distintos países.

Finalmente, el tercer postulado denominado posición relativa [Diener et al. (2002)] tiene relación con que "se puede ser muy feliz", siempre y cuando "el de al lado esté menos feliz". La posición relativa se relaciona con ingresos relativos y no con ingresos absolutos, en base a siete determinantes: ingresos, trabajo de calidad, comunidad, capacidad social- libertad de elección, reciprocidad política, igualdad y, sensación de bienestar. Dentro de este último postulado, se desprende un elemento fundamental y que abarca en completitud el real alcance que se puede dar en políticas públicas a este tema.

\section{Mediciones de Felicidad en Chile}

El Reporte Mundial de Felicidad (World Happiness Report) -de los economistas Heliwell, Layard y Sachs (20I2)- ha puesto varios años de investigación dando una figura bastante clara por sobre la simple intuición. Por ejemplo, en el ranking de felicidad emocional de afectos positivos (orgullo, goce y risas el día anterior) de la Encuesta Gallup Mundial $201 \mathrm{I}$, Chile se ubica en la posición $4 \mathrm{I}$ de 156.

Sin embargo, cuando nos referimos a los afectos negativos (preocupación, tristeza, enojo y depresión) Chile cae a la posición 136. Esto quiere decir que cuando a los chilenos se les pregunta sobre felicidad en el pasado (día anterior) las respuestas pueden variar absolutamente.

Al respecto, cabe destacar el reciente Informe sobre Desarrollo Humano en Chile publicado por el Programa de las Naciones Unidas para el Desarrollo (PNUD) este año. El Informe 2012 coloca especial énfasis en una versión más amplia de la felicidad: bienestar subjetivo. No hay que entender la felicidad únicamente como la satisfacción que tienen las personas con sus vidas, también hay que incluir la evaluación que hacen de la sociedad en que viven.

Al explorar la relación entre bienestar subjetivo individual y con la sociedad se puede analizar que hay un grupo mayoritario en la población chilena: "satisfechos y descontentos" (32\%). Esto indica que hay personas que declaran estar muy satisfechas con sus vidas, sin embargo están muy molestas con Chile \{las variables que incluye el nivel social tiene que ver con la "confianza de instituciones" y "evaluación de las oportunidades que entrega Chile para el desarrollo de capacidades"\}.

Muchas personas en Chile sienten que poseen capacidades (autoeficacia) pero no cuentan con los escenarios para desplegarlas. Esta interpretación cobra mayor fuerza si se considera que la situación es característica de los grupos de clase media alta y alta, que en términos subjetivos abarcan a gran parte de la población. Han alcanzado niveles de capacidad suficiente para imaginar proyectos de vida y comenzar a desarrollarlos (como el de iniciar un nuevo negocio), pero en el camino se han encontrado con obstáculos, como el no reconocimiento, que han producido en ellas un profundo malestar con la sociedad (lo que quiere reflejar la variable actitud en esta investigación).

\section{Antecedentes del modelo basado en la Intención de Emprender (IE)}

Shapero (1982) propuso uno de los primeros modelos teóricos acerca de las intenciones de emprendimiento. Su modelo introdujo el concepto de "Desplazamiento", entendido como un evento que, en conjunto con otros factores personales, cambia la ruta de las acciones que provocan el nacimiento de un nuevo negocio. Hay dos aspectos importantes que, en presencia de un acontecimiento vital desencadenante, puedan influir en la intención de crear un negocio: deseabilidad y viabilidad. El primero hace referencia al deseo por parte de los individuos de comportarse como emprendedores, $y$ el segundo, también entendido como factibilidad, hace mención a la confianza que las personas pueden tener al hacer un buen trabajo como emprendedores. En la presencia de un "desplazamiento", es altamente probable que las personas tengan la intención de emprender un negocio [Shapero (1982)].

Incluso en un evento desencadenante en la vida, deseabilidad y viabilidad son necesarios para disponer de los individuos hacia el comportamiento. Shapero (1982) examinó el concepto de deseabilidad usando información acerca de la familia y redes sociales [networking] de potenciales emprendedores. Se asumió que la variable familia, entendida como el contexto cultural para desarrollo de habilidades emprendedoras, afectaría positivamente hacia una actitud de convertirse en emprendedor.

Otra contribución importante de Shapero (1982) fue el haber considerado a la habilidad individual de identificar y obtener información financiera como un predictor importante de viabilidad. Shapero fue enfático en señalar que la percepción de tener acceso al dinero incrementaba la percepción de viabilidad. En esta línea, hay varios estudios que estudian de manera directa $o$ indirecta la influencia de variables financieras y económicas en la intención de emprender un negocio. Desde la percepción sobre la situación económica del país al momento de emprender [Serida y Morales (20II), lakovleva et al. (20II)] hasta el acceso a fuentes de financiamiento y niveles de disponibilidad de in-

ISSN: 07 I8-2724. (http://www.jotmi.org)

Journal of Technology Management \& Innovation @ Universidad Alberto Hurtado, Facultad de Economía y Negocios. 
gresos monetarios del individuo cuando están tomando la decisión de comenzar un nuevo emprendimiento [Fini et al. (2009)].

Por otro lado, Krueger y Carsrud (1993) argumentaban que la percepción de viabilidad de Shapero era similar al concepto de autoeficacia de Bandura (1986). La autoeficacia es un constructo ampliamente probado en la literatura psicológica como antecedente del comportamiento planificado y hace referencia a la creencia estable de la gente sobre su capacidad para manejar adecuadamente una amplia gama de estresores de la vida cotidiana. Pareciera ser que la autoeficacia tiene una importante relación con la intención de emprender. En efecto, varios estudios sugieren que la autoeficacia tiene un impacto en la intención de emprender y en el desempeño empresarial [Buam et al. (200I), Hmieleski y Baron (2008)]. Los individuos con altos niveles de autoeficacia son más probables que tengan un alto nivel de persistencia, concentración y comportamiento satisfactorio en el trabajo y por lo tanto un alta probabilidad que emprendan un nuevo negocio y su resultado sea el esperado. La intención de comenzar un negocio con la intención de emprender (IE) es utilizada como sinónimo en la literatura especializada.

La teoría del comportamiento planificado - TCP - [Ajzen (199I)], que es una extensión de la teoría del comportamiento razonado desarrollada por Fishbein y Ajzen (1975), explica la complejidad de la relación entre el comportamiento humano y sus determinantes, identificando el comportamiento humano como una causa de la intención. La TCP postula tres conceptos independientes que son determinantes en la intención, agregando un constructo que Shapero (1982) describió como: actitudes hacia el comportamiento, normas subjetivas, y percepción de control del comportamiento. Una actitud hacia el comportamiento se refiere al grado en el cual la persona desea comportarse en alguna forma. Normas subjetivas son entendidas como la percepción de la presión social para realizar un comportamiento,y de manera particular la percepción de control relacionado con la percepción de dificultad de la realización del comportamiento.

Ésta última se asume que refleja la experiencia previa así como también los impedimentos y obstáculos anticipados. En otras palabras, los tres factores mencionados afectan directamente la intención de una persona para tener una conducta específica. Según Ajzen (199|), mientras más favorables es la actitud y las normas subjetivas con respecto a un comportamiento, $y$ mayor es la percepción de control del comportamiento, más fuerte debe ser una intención individual para realizar una acción en específico. Esta teoría ha tenido una amplia variedad de aplicaciones.

Por ejemplo, en el estudio del comportamiento del consum- idor respecto a la intención de comprar o no un bien, comportamiento de las personas respecto al ocio, respecto al aumento el número de hijos, comportamientos respecto a la intención de beber alcohol, entre otras [Koe et al. (20I2)]. Krueger y Carsrud (1993) complementaron la teoría del comportamiento planificado con el evento emprendedor de Shapero, ofreciendo un modelo revisado sobre las intenciones emprendedoras. Kruger y Brazeal (1994) propusieron que el deseo y la autoeficacia son los predictores más importantes de la intención emprendedora, relegando la relevancia de las normas sociales a un segundo escalón. Afirmaron que las personas canalizan sus deseos y talentos hacia la percepción de una oportunidad, en base a creencias internas de eficacia y deseabilidad. Por tanto, la intención emprendedora promueve o inhibe la identificación de una oportunidad que es antecedida por cualquier acción emprendedora.

Krueger at al. (2000) también sugirieron la presencia de los grados de intención, los cuales pueden variar dependiendo de cuan fuerte sea la intención. Es más probable que, en el futuro, las intenciones débiles cambien más que las fuertes, $y$ por lo tanto el emprendimiento es el resultado de un comportamiento intencionado y planeado.

Una amplia literatura documenta el rol que tiene el capital humano, incluida la educación y los activos de conocimiento, pero la magnitud del impacto que tiene en todo el proceso de emprendimiento aún no ha sido debidamente consensuado desde el punto de vista académico [Unger et al. (20lI)]. El canal de transmisión de la educación en el emprendimiento tiene al menos tres dimensiones.

La primera se refiere a la capacidad de enfrentar y desempeñar nuevas tareas y oportunidades de negocios lo que implica tener un mayor nivel de educación [Shane and Venkatraman (2000)]. La segunda implica que el capital humano está positivamente relacionado con la planificación y la estrategia de emprendimiento [Frese et al (2007)], y la tercera es que el capital humano facilita la adquisición de capital físico y financiero [Brush et al. (200I)].

Asimismo, la influencia del estatus familiar socioeconómico en el desarrollo de los individuos y la intención de emprender, tales como logros académicos y desarrollo de carrera, son explicados por el nivel de educación [Bradley y Corwyn (2002); Porfeli y Vondracek (2005)]. Un efecto similar del status familiar socioeconómico también ha sido documentado en investigación sobre liderazgo, la cual muestra, por ejemplo, que la ocupación influye en la capitalización de recursos financieros.

Estos recursos pueden estimular un aprendizaje y desarrollo cognitivo [(Bass y Bass (2008)], aumentar el acceso a opor- 
tunidades de trabajo y capital social (Bradley et al. (200I)], los cuales debieran llevar a los individuos a tener la intención de iniciar nuevos negocios, dado el interés por desarrollar habilidades de liderazgo.

Baum et al. (200I) desarrollan un modelo multidimensional usando ecuaciones estructurales para explicar la tasa de crecimiento de un negocio. El modelo contiene 8 factores, entre los que se encuentra aquellos asociado a la personalidad de los individuos incluyendo variables como tenacidad, pasión, proactividad y factores motivacionales además de variables relacionadas a la visión y la orientación a objetivos, pero la variable de percepción de felicidad no se estudia en su modelo, y tampoco se menciona.

Los experimentos de Uy et al. (20I2) consideran implícitamente a la felicidad desde una perspectiva del bienestar psicológico, analizando cómo los emprendedores nacientes tratan de evitar afrontar situaciones estresantes al comenzar un negocio. Si bien demuestran una relación positiva entre bienestar sicológico y menor afrontamiento, no hay ninguna luz del impacto que tiene la felicidad. Baron y Tang (20II) concluyen que las personas que preponderan emociones positivas tienden a ser más exitosas y cumplir múltiples logros en distintos dominios de la vida, no sólo porque el éxito lleva a la felicidad, sino porque el afecto positivo engendra éxito.

No obstante, nunca dejan en claro si dentro de ese afecto positivo está el componente de felicidad, aunque eso se podría explicar por el hecho que la consideran una variable endógena. Cabe destacar el estudio presentado por Patzel y Sheperd (20II), en donde demuestran que a pesar de que los trabajadores por cuenta propia (como iniciar un nuevo negocio) pueden ser considerado el "lado oscuro del emprendimiento" [Kets de Vries (1985)] por las emociones negativas que conlleva (estrés, miedo, soledad, entre otras), estos en promedio, presentan menos emociones negativas que aquellos que están empleados. En el análisis estadístico de un cuestionario aplicado a 2700 personas de Estados Unidos los lleva a respaldar sus argumentos, agregando además que este efecto es contingente sobre el uso de las personas tanto de la concentración de los problemas y emociones, en los enfoques de afrontamiento de situaciones adversas. Cardon et al. (2012) atribuyeun completo nuevo concepto denominado Pasión Emprendedora (PE), afirmando ésta puede fomentar la creatividad $y$ el reconocimiento de nuevos patrones de información, y por consiguiente, nuevas oportunidades. No obstante, PE no es considerada como variable cognitiva, sino como variable explicada.

Foo (20I I) explica la felicidad en una dimensión espléndida: baja percepción de riesgo debido a tendencias de evaluación de alta certidumbre y control individual. Es decir, una perso- na feliz tiene la capacidad de evaluarse a sí misma con mayor frecuencia, lo que a su vez la llevaría a ver mejores futuros, y por tanto, a tener una mayor intención a emprender. En paralelo, no hay que dejar de lado una discusión e implicación práctica con respecto al Afecto Positivo de la Disposición o DPA por sus siglas en inglés (Dipositional Positive Affect), definido como la tendencia estable de presentar estados de ánimos y emociones positivas, el cual efectivamente puede beneficiar a las personas que lo experimentan [Baron et al. (20I2); Diener et al. (2002)].

Sin embargo, es importante considerar que especialmente a altos niveles de DPA, la sensación de estar "arriba" (con tanto entusiasmo y optimismo), puede llevar a creer que el hecho de empezar un nuevo negocio, la intención de emprender exitosamente, "es mucho para tanta cosa buena" [Grant y Schwartz (20II)]. Por lo tanto, la sugerencia es ser cauteloso con los afectos positivos, en donde los individuos deben ser capaces de maximizar los potenciales beneficios pudiendo hacer convergencia, eventualmente, con altos niveles de DPA.

Una atención específica a esta posibilidad pareciera ser un paso útil desde el punto de vista de ayudar a los emprendedores a alcanzar el éxito que tanto desean, lo que nos lleva a pensar que: $(I)$ en principio, habría una correlación entre felicidad y autoeficacia, y (2) que al mismo tiempo la felicidad, si la alejamos del concepto de DPA, puede ser un predictor directo de la intención emprendedora, invitándonos a extender los modelos de emprendimiento de Shapero y Ajzen, $y$ en concordancia con el planteamiento de Elfing et al. (2009).

Por otro lado, el efecto de la deseabilidad y la autoeficacia ha sido tratado por Fitzsimmons y Douglas (20II) mostrando que a menores niveles de deseabilidad, las personas pueden transformar la intención en un acto de emprender si es que ellas perciben que tienen la suficiente percepción de factibilidad (conocimiento) para hacerlo, contrastando a Shapero (1982). Pero también descubren evidencia empírica que individuos con fuertes percepciones de deseabilidad puede tener intención emprendedora incluso cuando perciben que no tienen la suficiente factibilidad (autoeficacia) para hacerlo. Este hallazgo nos lleva a cuestionar la semántica al referirnos a viabilidad, factibilidad y autoeficacia como sinónimos.

Dado los estudios anteriores, este trabajo no incorpora el ingreso ni el contexto económico de las personas, definido como apoyo y compensación (habilidad de obtener información) de debilidades desde el punto de vista financiero, dentro de viabilidad, y por consiguiente, no lo incluye en la dimensión de autoeficacia, sino que lo plantea como un predictor adicional que explique la intención emprendedora. Con esto se trata de reflejar que cada constructo que se 
relaciona con el emprendimiento se construye de manera independiente [Kuratko (2007)].

El modelo que guía las hipótesis de este estudio de incluir la variable felicidad, está inspirado en las recomendaciones de Casrud y Brannback (20II), y en la invitación de Elfing et al. (2009) de ajustar el modelo estándar.

Elfving et al. (2009) plantean un cambio en la forma lineal de mirar la intención de emprender proponiendo un modelo contextual general que sin perder los fundamentos centrales que proporcionan las teorías existentes, conecta factores motivacionales, de autoeficacia e intención, y de manera particular direcciona el rol de los objetivos específicos de los individuos y la motivación en el rol de emprender, además de mostrar la inconsistencia que tienen las normas sociales en el rol de emprender. Este trabajo de alguna forma invita y abre un espacio para que de manera fundada se le agreguen/ eliminen variables o factores a los "modelos estándar".

Carsrud y Brannback (20II), además de realizar una extensa, actualizada, crítica y ordenada revisión de literatura acerca de la intención de emprender, plantea 26 importantes preguntas de investigación acerca de lo que se necesita aún saber para explicar los determinantes que llevan a los individuos a tener la intención de emprender un nuevo negocio. Si bien no plantea la felicidad de manera explícita como una variable a estudiar, cuando propone la inclusión indirecta de los factores que explican la motivación para emprender se interpreta una causalidad potencial para esta variable.

Acorde a lo que se muestra en el modelo presentado y que está basado en revisión de literatura, en la presente investigación se plantean las siguientes hipótesis:

HI: Los individuos más felices tienen una mayor intención de emprender.

$H 2$ : Hay una interacción positiva entre felicidad y autoeficacia, de manera que la felicidad afecta indirectamente la intención de emprender.

\section{Método}

Se utilizó un método de investigación cuantitativo donde se analizó una muestra de 7.195 adultos de nacionalidad chilena. Sin embargo, la muestra con la que se trabajó finalmente fue

\footnotetext{
a Todas las personas que respondieron que no tenían intención de emprender (valor "0" para la variable FUTNEG) fueron analizadas, eliminándose si respondían afirmativamente (valor "I") en alguna de las siguientes variables: ESTBBUSO (Establecidos), TEAII (Emprendimiento en Etapas Iniciales), OWNMGE (Dueños o Administradores), y DISCENT (Descontinuados).
}

de 3.958 dado que se realizó un filtro de la variable dependientea. Se considera una muestra únicamente de aquellas personas que no tienen negocio establecido, que no están emprendiendo en etapas iniciales (TEA), ni tampoco los que han descontinuado o son dueños de negocios. Los análisis estadísticos se hicieron usando STATA/IC II.2. A continuación se detalla los métodos de recolección de datos, las variables analizadas, $y$ el modelo econométrico.

\section{Muestra y recolección de Información}

La fuente de información es el Global Entrepreneurship Monitor (GEM). El proyecto GEM Chile se inició en 2002 y es parte del proyecto GEM Mundial del cual han participado más de 80 países. Este proyecto tiene por objeto medir la actividad emprendedora y sus condicionantes, buscando identificar áreas de oportunidad para la aplicación de políticas públicas eficientes en promover el emprendimiento.

El reporte GEM recopila información primaria de la Encuesta de Población Adulta (Adult Population Survey, APS), la cual consideró una muestra de entrevistados entre 18 y 64 años en Chile durante los meses de mayo a agosto del año $20 \mathrm{II}$. El GEM recolecta información a través de un cuestionario aplicado telefónicamente. En esta encuesta se hacen preguntas precisas acerca de la participación en -y la actitud haciael emprendimiento. La encuesta utilizó muestreo aleatorio estratificado para localizar a los adultos en hogares, acorde a los lineamientos estrictos establecidos por el GEM y supervisados por los administradores de datos internacionales del GEM (Reynolds et al., 2007; Levie, 2008). El año 20I I, el GEM introdujo un conjunto adicional de preguntas en la APS con el fin de medir el rol de la felicidad en las diferentes etapas del proceso emprendedor y capturar la información específica acerca de la población total.

\section{Variables independientes, de control y dependiente}

Las variables que componen los factores relacionados con Actitudes, Autoeficacia y Contexto Económico, a excepción de Felicidad (variables independientes) han sido teóricamente identificados como predictores de intención emprendedora (variable dependiente). La intención es el principal antecedente de la acción emprendedora [Ajzen (I99I)] y como tal es importante conocer que variables pueden predecir la intención de mostrar un comportamiento. En este caso el comportamiento de iniciar un negocio (emprender). Para lo anterior, se optó por elegir la primera fase de la actividad de emprender - debido a que se encuentra disponible una extendida literatura enfocada en el emprendimiento potencial -conocimiento y habilidades- [Amorós y Poblete (2012); Hessels et al. (2010)] como predictor de la intención de emprender. 


\section{Análisis}

De la muestra a investigar GEM, $43 \%$ de los entrevistados son hombres y tienen un promedio de edad de $4 \mathrm{I}$ años y 57 $\%$ son mujeres con promedio de edad de 44 años El $40 \%$ de las personas entrevistadas declararon que tenían la intención de comenzar un nuevo negocio en los próximos 3 años. Un $92 \%$ de los entrevistados se consideraban felices, mientras que el $81 \%$ se encontraba satisfecho con su vida. Además, el $20 \%$ de la muestra tiene al menos educación universitaria completa. Por ultimo, el $52 \%$ de las personas de la muestra cree que posee los conocimientos, habilidades y experiencia requerida para empezar un nuevo negocio (autoeficacia).

En esta investigación se utiliza un modelo LOGIT para verificar los efectos específicos y especialmente la dirección que tiene la felicidad en la intención de emprender de conformidad a las hipótesis planteadas. Conceptualmente, la idea de un LOGIT es que puede derivarse a partir de un modelo de regresión, en donde la variable dependiente es latente y sólo se observa una respuesta dicotómica de la misma. La variable observable representa la elección, por parte de los individuos, entre dos posibles alternativas. Por lo tanto, se modela una variable latente no limitada en su rango de variación, $V^{*}$. Cuando la variable latente supera un determinado nivel, la variable discreta toma el valor I, y si no lo supera toma el valor 0 . En otras palabras, la variable $\gamma^{*}$ representa el "sentimiento" o "deseo" del individuo hacia una de las alternativas pero, en la práctica, lo único observable es que dicho individuo elije esa alternativa o no. Si se denota $Y_{i}$ la realización dicotómica de $Y_{i}^{*}$ se puede establecer la siguiente relación:

$$
Y_{i}=\left\{\begin{array}{c}
1, \quad \text { si } Y_{i}^{*} \geq 0 \text { lo que ocurre si } X_{i k} \beta+\varepsilon_{i} \geq 0^{\mathrm{a}} \\
0, \quad \text { si } Y_{i}^{*}<0 \text { lo que ocurre si } X_{i k} \beta+\varepsilon_{i}<0
\end{array}\right.
$$

Donde se asume que el vector $X_{i}$ contiene las observaciones de $k$ variables explicativas del individuo i-ésimo, $\beta$ es un vector de coeficientes y $\varepsilon_{i}$ son variables aleatorias que se distribuyen como una curva logística [Green (2003)]. Lo que nos interesará para el análisis LOGIT, dada las dos hipótesis planteadas será el signo de los parámetros, lo que indicará la dirección en que se mueve la probabilidad de la intención de emprender cuando cambia la variable explicativa correspondiente. En función del supuesto de distribución logística para el término aleatorio $\varepsilon_{i}^{b}$, el modelo se puede expresar como sigue, donde se asegura que los valores de $\gamma_{i}$ tomen valores entre 0 y I:

\footnotetext{
${ }^{a} X_{i k} \beta+\varepsilon_{i} \geq 0$ corresponden a las "ganancias netas de emprender". b La función de distribución logística es: $F(X)=$ $\frac{e^{x}}{1+e^{x}}$
}

$$
Y_{i}=\frac{e^{\beta_{0}+X_{k i} \beta_{k}}}{1+e^{\beta_{0}+X_{k i} \beta_{k}}}+\varepsilon_{i}=\frac{1}{1+e^{-\beta_{0}-X_{k i} \beta_{k}}}+\varepsilon_{i}
$$

Si representamos como $p$ a la probabilidad de que ocurra el evento, entonces alternativamente el modelo logístico puede ser representado como sigue:

$$
\ln (\mathrm{p})-\ln (1-\mathrm{p})=\ln \left(\frac{p}{1-p}\right)=\beta_{0}+X_{k i} \beta_{k}+\varepsilon_{i}
$$

Obsérvese que el modelo LOGIT, es decir la diferencia de la probabilidad de que ocurra un suceso respecto de que no ocurra es lineal pero en escala logarítmica. De esta forma, el modelo a estudiar considerando las variables definidas en la tabla I se puede expresar como sigue:

$$
\mathrm{Y}=\ln \left(\frac{p}{1-p}\right)=\beta_{0}+\sum_{k=1}^{9} \beta_{k} X_{k}+\sum_{k=1}^{3} \gamma_{k} Z_{k}+\varepsilon_{i}
$$

Dónde $X_{k}$ es una variable explicativa, $Z$ es una variable de control. La variable $Y$ es la variable que mide la probabilidad de la intención de emprender. Complementariamente, para medir los efectos de moderación de la felicidad (variable $X_{8}$ y variable $\left.X_{9}\right)$ sobre el factor de autoeficacia $\left(X_{1}\right)$ se construyeron dos variables adicionales: la variable $X_{10}=X_{8}$ $X$ que considera el efecto moderación de la declaración de felicidad del individuo sobre la variable $X_{1} y ; X_{11}=X_{9} X_{1}$, la cual considera el efecto moderación de la escala de satisfacción con la vida en $X_{1}$.

El parámetro de la variable $X_{10}$ y la variable $X_{11}$ corresponden al efecto de moderación de la felicidad (en las dos medidas de felicidad) con respecto a autoeficacia. La dirección del efecto de autoeficacia en la intención de emprender se mide a través de los signos del parámetro asociado a la variable $X_{1}$ y la variable $X_{10}$ y $X_{11}$, es decir se incluye una variable dicotómica en "pendiente" pero de diferencias de logaritmos de probabilidad.

Es importante dejar en claro que el uso de este modelo tiene como propósito analizar las variables que influyen en la probabilidad que un individuo elija un curso de acción: intención de emprender. Dado que no se intenta predecir, los coeficientes estimados solamente permiten contrastar la dirección de los signos esperados a priori. No se interpretan utilizando efectos marginales.

Como tal, el modelo LOGIT al no ser lineal, no es posible aplicar mínimos cuadrados y en consecuencia, se debe aplicar el método de máxima verosimilitud (ML) [Cristi (20/2)]. La estimación de máximo verosimilitud busca aquellos valores de los parámetros que generarían con mayor proba-

ISSN: 07 I8-2724. (http://www.jotmi.org) 
bilidad la muestra observada. El signo de los coeficientes indica la dirección en que se mueve la probabilidad de intentar emprender al cambiar alguna de las variables explicativas.

\section{Resultados}

La hipótesis I, que indica que la felicidad tiene un efecto positivo en la intención emprendedora, no pudo ser comprobada en las distintas regresiones LOGIT que se realizaron. En efecto, la felicidad aproximada en escala de satisfacción de vida del individuo $\left(X_{q}\right)$ resultó tener un signo negativo y significativo distinto de cero al $99 \%$, mientras que el signo de la variable $X_{8}$ es negativo y significativo al $90 \%$.

Asimismo, la hipótesis 2 tampoco fue comprobada. La variable felicidad, definida en escala de satisfacción con la vida $\left(X_{I I}\right)$ modera de manera negativa ( $y$ no positiva) en la dirección que implica la autoeficacia en la intención de emprender, en la forma como se ha aproximado a través de la variable $X_{1}$.

En lo que sigue se muestran los análisis en diferentes modelos testeados, los que permiten concluir los resultados anteriores.El modelo directo considera una regresión LOGIT, el cual selecciona variables específicas para cada constructo conceptual (Autoeficacia, Actitud, Contexto Económico) e incluye la variable $X_{9}$ por $X_{8}$. Es decir, se considera la escala de satisfacción con la vida como representativa de la felicidad. El modelo indirecto incluye el efecto indirecto de moderación sobre autoeficacia para la $X_{8}$ y $X_{9}$ respectivamente. Ambos modelos se enmarcan son respaldados por la invitación de Elfving et al. (2009), agregando o eliminando nuevas variables al modelo estándar. Los resultados resumidos de los dos modelos LOGIT se presenta en Tabla 2.

La bondad de los ajustes para cada modelo puede ser verificada a través del test de LR. Las estimaciones muestran que todos los modelos hay una adecuada bondad de ajuste expresado por el test de la razón de verosimilitud (LR: modelo restringido vs modelo no restringido) ${ }^{*}$. En ambos modelos estimados, la felicidad muestra un signo negativo. En el modelo directo se trabaja con una sola variable explicativa para cada uno de los tres constructos teóricos (autoeficacia, actitud y contexto económico), y se incluye la variable $X_{9}$ en cada una de las regresiones. Los parámetros de las tres variables "teóricas" $X_{1}, X_{3} X_{6}$ son significativos distintas de cero y con los signos esperados. Es decir un mayor nivel de conocimiento de las habilidades del individuo, deseabilidad de iniciar una carrera de emprendimiento y apoyo económico tienen individualmente efectos positivos en la probabi-

\footnotetext{
* Se define como

$$
-2 \ln \frac{L\left(\widetilde{\beta_{R}}\right)}{L\left(\widetilde{\beta_{N R}}\right)} \sim x_{r}^{2}
$$
}

lidad de iniciar un emprendimiento. El signo de la variable felicidad medida por la variable $X_{q}$ es negativa y altamente significativa (99\%).

En el modelo indirecto se observa a través del signo de la variable $X_{\| l}$, que la felicidad medida como escala de satisfacción con la vida modera el efecto autoeficacia a través de la variable $X$, lo que se determina por el valor negativo y significativo del parámetro. Lo anterior indicaría que el efecto de la autoeficacia en la probabilidad de la intención de emprender es menor en los individuos felices.

En todos los modelos la variable sexo resultó ser no significativa. La variable educación resulto ser significativa en el modelo indirecto. La variable edad resultó ser significativa en los dos modelos. En la siguiente figura se representa de manera aproximada la dirección del efecto indirecto, a través del cambio de "pendiente" de la relación entre la probabilidad de intención de emprender y la variable que aproxima autoeficacia, bajo el entendido que el efecto marginal en un LOGIT no es la pendiente. Cuando los individuos no son

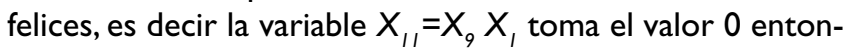
ces la probabilidad de la intención de emprender cuando la autoeficacia se incrementa es mayor que cuando la variable $X_{11}$ toma valor $\mathrm{I}$.

Cuando los individuos no son felices, es decir la variable $X_{11}=X_{9} X_{1}$, toma el valor 0 entonces la probabilidad de la intención de emprender cuando la autoeficacia es mayor que en el caso cuando la variable toma valor I.

\section{Discusión}

En este trabajo se ha examinado el efecto de la felicidad en la intención emprendedora, y no el efecto del emprendimiento en la felicidad ${ }^{\text {a }}$ Para lo anterior, se realizó una revisión de literatura y se ha construido un modelo uni-ecuacional estimado a través de un LOGIT. El hallazgo más importante es que, bajo diferentes aproximaciones para la variable felicidad,

\footnotetext{
${ }^{a}$ En principio para el caso de Chile, si bien no hay evidencia científica que el emprendimiento tiene un efecto positivo en la felicidad de las personas, a nivel macroeconómico Schmidt-Hebbel (20I2) señala que dado el nivel de ingreso per cápita alcanzado por el país, explicado principalmente por el emprendimiento, entonces los chilenos están más felices comparativamente a otros países.

Asimismo, Amorós (201 I), a la luz de una serie de acontecimientos ocurridos en el país el año $201 \mathrm{I}$, se pregunta si ipueden los emprendedores contribuir a la felicidad? y opina que los emprendedores sociales han logrado traspasar la felicidad a mucha gente, así como empresarios exitosos se han dedicados a traspasar a "través de bienes materiales un mayor bienestar a la sociedad".Al respecto, ver sección siguiente sobre propuestas de futuras líneas de investigación.
} 


\begin{tabular}{|c|c|c|}
\hline & $\begin{array}{l}\text { Modelo Directo } \\
\mathrm{LR}=618.99 \\
\text { Pseudo } \mathrm{R}^{2}=0.1155\end{array}$ & $\begin{array}{l}\text { Modelo Indirecto } \\
\mathrm{LR}=617.49 \\
\text { Pseudo } \mathrm{R}^{2}=0.1152\end{array}$ \\
\hline \multirow{2}{*}{$\begin{array}{l}\text { Variable } \\
\qquad \begin{array}{l}X_{1} \\
X_{2}\end{array}\end{array}$} & $1.25(17.43)^{*}$ & $1.5590(11.71)^{*}$ \\
\hline & & \\
\hline $\begin{array}{l}X_{3} \\
X_{4}\end{array}$ & $.1089(1.29)$ & $.1094(1.30)$ \\
\hline$X_{5}$ & & \\
\hline$X_{6}$ & $.4638(4.22)^{*}$ & $.4658(4.24)^{*}$ \\
\hline$X_{7}$ & & \\
\hline$X_{8}$ & & \\
\hline$X_{9}$ & $-.2902(-3.12)^{*}$ & \\
\hline$X_{10}$ & & \\
\hline$X_{11}$ & & $-.3807(2.85)^{*}$ \\
\hline$Z_{1}$ & $-.1050(-1.47)$ & $-.1039(-1.46)$ \\
\hline$Z_{2}$ & $-.0029(-0.16)^{*}$ & $-.0070(0.40)$ \\
\hline$Z_{3}$ & $-.0329(-14.62)^{*}$ & $-.0327(-14.54)^{*}$ \\
\hline$\beta_{0}$ & $.6424(3.07)$ & $.4200(2.14)$ \\
\hline
\end{tabular}

Tabla I: Estimaciones LOGIT Nota: Los paréntesis incluyen el valor de z. *: refleja la significancia a 95\%.

ésta muestra una relación inversa con la intención de emprender, siendo más significativa cuando la felicidad se mide en escala de satisfacción con la vida. Esto nos lleva a constatar que individuos que manifiestan tener mayor grado de felicidad o que están más satisfechos con la vida tienen una menor probabilidad de poseer una intención de emprender. Lo anterior puede ser interpretado en dos direcciones: (a) Dado que los chilenos se sienten menos felices, tienen mayor intención de emprender, y de alguna forma emprenden en busca de la felicidad. (b) Una vez que los chilenos van alcanzando un mayor nivel de felicidad, es probable que su intención de emprender sea menor.

El primer punto podría ser coherente con el hecho mas general que de acuerdo a los reportes anuales del GEM, en Chile la tasa de emprendimiento en etapas iniciales (TEA) se ha incrementado en los últimos años (por ejemplo, el 2007 era igual a $13.43 \%$ y el 201 I fue igual a $23.70 \%$ ) y a la vez según el Informe de Desarrollo Humano del PNUD (20I2) el promedio de la percepción general de satisfacción con la vida de los chilenos estadísticamente ha sufrido una caída significativa desde el año 1990 al año 201 I $^{\text {a }}$

\footnotetext{
a Es atingente mencionar un reciente artículo publicado a comienzos de octubre 2012 por John Rathobe de la revista Financial Times: Se considera a Chile como un país infeliz, ya sea por el bienestar subjetivo o por malestar social.
}

Respecto al segundo punto, es sorpresivo haber encontrado una relación negativa entre intención de emprender y felicidad. La intuición inicial indicaba lo contrario. No obstante, si revisamos la conferencia de Gilbert (2004) se puede entender que la felicidad puede ser sintetizada. De acuerdo a Gilbert (2004) la felicidad natural va ligada a lo que "tenemos cuando obtenemos lo que queríamos". En cambio, la felicidad sintética es lo que "hacemos cuando no tenemos lo que queríamos". En su libro "Stumbling on Happiness", Gilbert indica que eventos traumáticos que afectan a la gente (como puede haber sido el terremoto 27F, y las protestas estudiantiles en Chile), sugiere que si sucedió hace más de 3 meses, con pocas excepciones, no tienen ningún efecto en la felicidad. Asimismo, al relacionar esta idea a la tendencia de sobrevalorar las diferencias de los futuros posibles, entonces se puede concluir que las preferencias "nos arrastran tan fuerte y demasiado rápido que no tienen limites". La paradoja indica que cuando las preferencias y miedos están limitados (o son casi irreversibles), nos lleva a ser más felices. Esto podría explicar de alguna manera la razón por la cual personas más felices en Chile tienen menor intención emprendedora. Son felices sintéticamente. Saben que no van a obtener lo que quieren, ya que expresan un alto grado de malestar social. Es decir, no vislumbran una institucionalidad confiable y eficiente que les permita tener un espacio para iniciar un nuevo negocio. Pero eso no los condiciona para ser felices, ya que más allá de las oportunidades, para los chilenos ser felices depende de ellos mismos. ¿O será que

ISSN: 07 I8-2724. (http://www.jotmi.org) 
emocionalmente se sientan presionados a responder que son felices?

\section{Limitaciones del Estudio}

Al trabajar con un modelo uni-ecuacional, las variables que se introducen son aproximaciones a los constructos principales que explican la intención de emprender de acuerdo a la teoría de Shapero (1982) y Ajzen (1991). En efecto, las variables principales soportadas por las teorías del emprendimiento constituyen variables latentes, es decir son no observables o medibles y solamente pueden ser explicadas y medidas por un conjunto de variables observables, por ejemplo a través de un cuestionario. Un ejemplo, es la variable actitud $y / o$ la variable autoeficacia. Ambas variables son latentes y se explican por otras variables de un cuestionario.

El modelo presentado no incluye las variables relacionadas a Normas Sociales, las que si tienen amplia justificación desde el punto de vista de la teoría. Estas variables están incluidas en la Encuesta Nacional de Expertos (National Experts Survey, NES), pero no en la APS, y ambas encuestas no pueden ser combinadas desde el punto de vista estadístico (distinto tamaño muestral y tipo de estudio).

\section{Implicancias para futuras líneas de investigación}

Resultaría de sumo interés como línea de investigación agregar un conjunto de preguntas adicionales al cuestionario del GEM, que permitiera explicar los determinantes de las variables que afectan la felicidad, y de esta forma generar un constructo más completo sobre ella, de tal forma de re-testear los resultados y efectos en la intención de emprender.

También para profundizar los resultados, resultaría apropiado estudiar las otras fases del proceso de la actividad emprendedora, en la forma como las define el GEM. Adicionalmente, un estudio comparativo usando un panel de países es otra línea de investigación que se abre para verificar los efectos de la felicidad en la intención emprendedora. Finalmente, resultaría interesante establecer un análisis factorial exploratorio y confirmatorio bajo la forma de ecuaciones estructurales. Por ejemplo, si del análisis exploratorio se verificara que las variables se agrupan como se muestra en la tabla siguiente, entonces sería posible establecer un modelo de ecuaciones estructurales. Esto con el objetivo de confirmar y establecer la dirección de los efectos en un modelo muldimensional y no solo uni-ecuacional como el caso del LOGIT estimado.

\section{Referencias}

ACHOR, S. (2010), The Happiness Advantage: The Seven Principles of Positive Psychology That Fuel Success and Performance at Work Crown Publishing Group, 2010.

ACS, Z.J., Arenius, P., Hay, M. y Minniti, M. (2004), Global Entrepreneurship Monitor: 2004 Executive Report, Babson College, Babson, MA.

AJZEN, I., y M. Fishbein (I980), Understanding attitudes and predicting social behavior. EnglewoodCliffs, NJ: Prentice-Hall.

AJZEN, I. (199I) "The theory of planned behavior". Organizational Behavior and Human Decision Processes, 50(2), |79-2|I.

ALESINA, A., Di Tella, R., y R. MacCulloch (2004) "Inequality and happiness: are Europeans and Americans different?" Journal of Public Economics, 88(9-10), 2009-2042.

AMÓROS, J.E. y Cristi, O. (20I I). Poverty, Human Development and Entrepreneurship, In Minniti, M. (Ed.) The Dynamics of Entrepreneurship: Theory and Evidence. Oxford: Oxford University Press, pp. 209-230.

AMORÓS, J.E. y Poblete, C. (2012) Reporte Nacional de Chile 2012 Global Entrepreneurship Monitor- GEM. Santiago: Universidad del Desarrollo.

AMORÓS, J.E. (20II) ¿Pueden los emprendedores contribuir a la felicidad? Guioteca Chile. Disponible en: http:// www.guioteca.com/emprendimiento/\%C2\%BFpueden-losemprendedores-contribuir-a-la-felicidad/ (publicado el 23 de septiembre, 20I I)

BANDURA,A. (1986), Social foundations of thought and action:A social cognitive theory. Englewood Cliffs,NJ: PrenticeHall.

BARON, R., y S. Shane (2005), Entrepreneurship:A process perspective (Ist edition). Mason, OH:South-Western Publishing.

BARON, R., Hmieleski, K. M., y R. Henry (20I2) “Entrepreneurs' dispositional positive affect:The potential benefits and potential costs - of being up". Journal of Business Venturing, 27(3).

BARON, A., y Tang, J. (20II) "The role of entrepreneurs in firm-level innovation:Joint effects of positive affect, creativity, and environmental dynamism". Journal of Business Venturing, 26(I), 49-60. 
BASS, B. M., y R Bass (2008), The Bass handbook of leadership: Theory, research, and managerial applications. New York: Free Press.

BAUM, J., Locke, E. y Smith, K. (200I). "A Multidimensional Model of Venture Growth. Academy of Management Journal". 44(2), 292-303

BEN SHAHAR, T. (20I2) Entrevista a Diario La Tercera. Santiago de Chile. Disponible en: http://diario.latercera. com/2012/05/06/0I/contenido/tendencias/ 16-107809-9-talben-shahar-ni-las-redes-sociales-ni-tener-cientos-de-amigos-en-facebook.shtmll (publicado el 6 de mayo, 2012)

BINDER, M. y T. BROEKEL (20I2) "Happiness no matter the cost? An examination on how efficiently individuals reach their happiness levels". Journal of Happiness Studies, 13 (4). Pp. 62I-645.

BINDER, M. (2012) "Innovativeness and Subjective Well-Being" Social Indicators Research. An International and Interdisciplinary Journal for Quality-of-Life Measurement Working Paper. Online ISSN I573-092I.

BJØRN, G. (2006), "Mental Health” in The Biology of Happiness. Netherlands: Springer.

BRADLEY, R. H., y R.F. Corwyn (2002) "Socioeconomic status and child development" Annual Review of Psychology, 53(I), 37I-399.

BRADLEY, R.H., Corwyn, R.F., McAdoo, H.P. y C Garcia Coll (200I). "The home environments of children in the United States: Part I,Variations by Ethnic and Income Group" Child Development, 72, 1844-1867.

BRUSH, C.G., Greene, P.G., y M.M. Hart (200I) "From initial idea to unique advantage: the entrepreneurial challenge of constructing a resource base" Academy of Management Executive 15, 64-78.

BUTKOVIC,A.; Brkovic, l.; Bratko, D. (20I2) "Predicting WellBeing From Personality in Adolescents and Older Adults" Journal of Happiness Studies Vol. I 3 (3): 4I I-437.

CAREE, M. y I.Verheul (20I2) "What makes entrepreneurs happy? Determinants of satisfaction amongst founders" Journal of Happiness Studies, I3(2), 37I-387.

CARDON, M. S., Gregoire, D. A., Stevens, C. E. y P. Patel (2012) "Measuring entrepreneurial passion: Conceptual foundations and scale validation" Journal of Business Venturing.

ISSN: 07 I8-2724. (http://www.jotmi.org)

Journal of Technology Management \& Innovation (c) Universidad Alberto Hurtado, Facultad de Economía y Negocios.
CARSRUD, A., y M. Brannback (20II) "Entrepreneurial Motivations : What Do We Still Need to Know ?" Journal of Small Business Management, 49(I), 9-26.

CHATURVEDI, S., Arvey, R., Zhang, Z. y Christoforou, P. (20II). "Genetic Underpinnings of Transformational Leadership:The Mediating Role of Dispositional Hope". Journal of Leadership \& Organizational Studies, 18(4), 469-479.

CRISTI, O. (20I2) "Apuntes de Clases: Econometría Financiera” MCA Santiago de Chile. Universidad del Desarrollo.

DIENER, E., Nickerson, C., Lucas, R.E. y E. Sandvik (2002) "Dispositional affect and job outcomes". Social Indicators Research, 59, 229-259.

DIENER, Lucas y Napa Scollon (2009), Beyond the Hedonic Treadmill: Revising the Adaptation Theory of Well-Being

EASTERLIN, R. (1974) "Does economic growth improve the human lot? Some empirical evidence". En P.A. David \& M.W. Reder (eds.), Nations and households in economic growth: essays in honor of Moses Abramovitz. New York \& London: Academic Press.

ELFVING, J., Brännback, M. y A. Carsrud (2009) "Toward A Contextual Model of Entrepreneurial Intentions" en Understanding the Entrepreneurial Mind. ed.A. L. Carsrud y M. Brännback, 23-33. New York \& London: Springer.

ETHUGALA, C.V. (20II) "Expectations of the Private and Civil Stakeholders Responsiveness of the State Sector: Tea Industry of Sri Lanka" Journal of APBITM I(I), I3-I9.

FINI, R., Grimaldi, R., Marzocchi, G.L. y Sobrero, M. (2009) "The Foundation of Entrepreneurial Intention", conference proceedings in Summer Conference 2009, June 17-19, 2009, Frederiksberg, Denmark.

FISHBEIN, M. y Ajzen, I. ( 1975). Belief,Attitude, Intention, and Behavior:An Introduction to Theory and Research. Reading, MA:Addison-Wesley.

FITZSIMMONS, J. R., y E.J. Douglas (20II) "Interaction between feasibility and desirability in the formation of entrepreneurial intentions" Journal of Business Venturing, 26(4), $431-440$.

FOO, M.D. (20II) "Emotions and entrepreneurial opportunity evaluation" Entrepreneurship Theory and Practice 35 (2), 375-393.

GLADWELL, M. (2003), The Tipping Point: How Little Things Can Make a Big Difference. New York: Little, Brown and Company. 
GRAHAM, C. y Shapiro, J. (2009) "Knowing Why Afghans Smile May Help Win Hearts and Minds" Disponible en http://www.washingtonpost.com/wp-dyn/content/article/2009/08/I0/AR200908/002456.html (publicado el 13 agosto, 2009)

GRANT, A.M., y B. Schwartz (20II) "Too much of a good thing: the challenge and opportunity of the inverted $U$ ". Perspectives on Psychological Science 6, 6I-76.

GREENE, W. (2003). Econometric Analysis ( $4^{\mathrm{a}}$ ed.), Prentice Hall. $3^{\mathrm{a}}$ edición en castellano:Análisis Econométrico.

GRETCHEN, R.(200I) "The Happiness Project: Or, Why I Spent a Year Trying to Sing in the Morning, Clean My Closets, Fight Right, Read Aristotle, and Generally Have More Fun" . Nueva York: HarperCollins

HARTUNG, P. J., Porfeli, E. J. y F.W.Vondracek (2005) "Child vocational development: A review and reconsideration" Journal of Vocational Behavior, 66(3), 385-4I9.

HELLIWELL, J., Layard, R. y J. Sachs, eds. (20I2), World Happiness Report, Nueva York: The Earth Institute, Columbia University.

HESSELS, J., I. Grilo, R. Thurik y P. van der Zwan (2010) "Entrepreneurial exit and entrepreneurial engagement". Journal of Evolutionary Economics,2I (3): 447-47I.

HMIELESKI, K.M. y R.A. Baron (2008) "When does entrepreneurial self-efficacy enhance versus reduce firm performance?" Strategic Entrepreneurship Journal 2,57-72.

IAKOVLEVA, T., Kolvereid, L., y U. Stephan (20I I). Entrepreneurial Intentions in Developing and Developed Countries, Education and Training, 53(5), p.353-370.

KETS DEVRIES, M. ( 1985 ) "The Dark Side of Entrepreneurship" Harvard Business Review, Vol. 63, Issue 6, p. 160-167 1985.

KEYNES, J. M. (1936). Teoría general de la ocupación, el interés y el dinero, Fondo, Cuarta edición (corregida y aumentada), 2003.México: Fondo de Cultura Económica.

KIM, S. y Kim D. (2012) "Does Government Make People Happy?: Exploring New Research Directions for Government's Roles in Happiness". Journal of Happiness Studies, 2012, vol. I3, issue 5, pp.875-899.

KRUGER, N.F. y A. Carsud (1993) "Entrepreneurship intentions: Applying the theory of planned behavior" Entrepreneurship Theory and Regional Development. 5: 315-330.
KOE, W.-L., Sa'ari, J. R., Majid, I. A. y K. Ismail (20I2) "Determinants of Entrepreneurial Intention Among Millennial Generation" Procedia - Social and Behavioral Sciences, 40, 197-208.

KRUEGER, Norris F. y D. Brazeal (1994) "Entrepreneurial Potential and Potential Entrepreneurs" Entrepreneurship Theory and Practice,Vol. 18, Issue 3, p. 91-104 1994.

KRUEGER, N.F. Jr., Reilly, M.D. y A.L Carsrud (2000) "Competing Models of Entrepreneurial Intentions" Journal of Business Venturing, 15:41 I-432.

KURATKO, D. F. (2007) "Entrepreneurial Leadership in the 2 Ist Century: Guest Editor's Perspective" Journal of Leadership \& Organizational Studies, I3(4), I-I I.

LAYARD, R. (2005), La felicidad: Lecciones de una nueva ciencia. Madrid:Taurus.

LARROULET, C. y J.P. Couyoumdjian (2009) “ Entrepreneurship and Growth:A Latin American Paradox" The Independent Review, v I4(I): 8I-100.

LASPITA, S., Breugst, N., Heblich, S. y Patzelt, H. (20I2). Intergenerational transmission of entrepreneurial intentions. Journal of Business Venturing, 27(4), 4I 4-435.

LYKKEN, D. (2006), The Heritability of Happiness. Harvard Mental Health Letter.

MEULMAN,J.J.y Heiser,W.J. (2005). SPSS Categories ${ }^{\circledR}$ I4.0.

NICOLAOU, N. y Shane, S. (2009). Can genetic factors influence the likelihood of engaging in entrepreneurial activity? Journal of Business Venturing, 24(I), I-22.

GILBERT, D. (2004), Stumbling on Happiness. Nueva York: Harper Collins Publishers.

PATZELT, H. y D. Shepherd (20II) "Negative emotions of an entrepreneurial career: Self-employment and regulatory coping behaviors" Journal of Business Venturing, 26(2), 226238.

PNUD (2012), Informe Desarrollo Humano en Chile: Bienestar subjetivo: el desafío de repensar el desarrollo. Santiago de Chile. Sinopsis disponible en: http://www.desarrollohumano.cl/

RATHOBE, J. (20I2) Protests: Middle class unhappy over continued inequalities. Financial Times. Disponible en: http://www.ft.com/intl/cms/s/0/53255400-03 I 0-I l e2-a28400 I44feabdc0.html\#axzz28vWrjCGJ (publicado el I octubre, 2012)

ISSN: 07 I8-2724. (http://www.jotmi.org)

Journal of Technology Management \& Innovation (C) Universidad Alberto Hurtado, Facultad de Economía y Negocios. 
RAUCH, A. y M. Frese (2006) "Meta-analysis as a tool for developing entrepreneurship research and theory" In: Wiklund, J., Dimov, D.P., Katz, J., Shepherd, D. (Eds.),Advances in Entrepreneurship, Firm Emergence, and Growth, Vol. 9. Elsevier, London, Pp. 29-52

SCHMIDT-HEBBEL, K. (20I2) El crecimiento económico en Chile. Diario El Mercurio, Economía y Negocios. Santiago de Chile. Disponible en: http://www.economiaynegocios.cl/noticias/noticias.asp?id=999। I (publicado el 28 de agosto, 20I2)

SHANE, S., y S.Venkataraman (2000) "The promise of entrepreneurship as a field of research"Academy of Management Review, 25(I), 217-226.

SHAPERO, A. (1982) "Social Dimensions of Entrepreneurship" Pp. 72-90 in C. Kent, D. Sexton and K.Vesper (eds.). The Encyclopedia of Entrepreneurship.

SELIGMAN, M. (20I2), Flourish:A Visionary New Understanding of Happiness and Well-being.New York: Simon \& Schuster, Inc.

SERIDA, J.y O. Morales (20II) "Using the theory of planned behavior to predict nascent entrepreneurship". Academia, Revista Latinoamericana de Administración, 46, 201 I, 55-7I

STEVENSON, B., y J.Wolfers (2008) "Economic Growth and Subjective Well-Being: Reassessing the Easterlin Paradox". SSRN Electronic Journal. Volume: 2008, Issue: I, Publisher: SSRN, Pages: I-87

THALER,R. y C. Sunstein (2009), Nudge: Improving Decisions About Health, Wealth, and Happiness. Nueva York: Penguin Books. [Un pequeño empujón (Nudge): El impulso que necesitas para tomar mejores decisiones sobre salud, dinero y felicidad, trad. de Belén Urrutia, México DF, Santillana]

UNANUE,W. (2012) Conferencia en $I^{\circ}$ Encuentro de Felicidad en Chile. Disponible en http://terratv.terra.cl/videos/ Entretenimiento/Vida-y-Estilo/Instituto-Felicidad-Coca-Cola/ (publicado el 28 de mayo, 20I2)

UNGER, J. M., Rauch, A., y M. Frese (20II) "Human capital and entrepreneurial success: Ameta-analytical review" Journal of Business Venturing , 34I-358.

UY, M., Foo, M. y Z. Song (2012) "Joint effects of prior startup experience and coping strategies on entrepreneurs psychological well-being" Journal of Business Venturing.

VAN DIERENDONCK, D. (20I2) "Spirituality as an essential determinant for the Good Life, its importance relative to self-determinant psychological needs" Journal of Happiness Studies, 13, 685-700.
WALLIS, C. (2005) The New Science of Happiness. Time Magazine. Disponible en: http://www.time.com/time/magazine/article/0,917/,I0I5832,00.html (publicado el 9 enero,2005) 